\title{
Basis Set Dependence of Optical Rotation Calculations with Different Choices of Gauge
}

\author{
Taylor Parsons, ${ }^{\dagger}$ Ty Balduf,,$^{\dagger}$ James R. Cheeseman, ${ }^{\ddagger}$ and Marco Caricato*, $\dagger$ \\ †Department of Chemistry, University of Kansas, 1567 Irving Hill Road, Lawrence, Kansas \\ 66045, United States \\ $\ddagger$ Gaussian, Inc., 340 Quinnipiac St., Bldg. 40, Wallingford, CT 06492, United States \\ E-mail: mcaricato@ku.edu
}

\begin{abstract}
In this work, the basis set dependence of optical rotation (OR) calculations is examined for various choices of gauge/level of theory. The OR is calculated for a set of 50 molecules using B3LYP and CAM-B3LYP, and 17 molecules using coupled cluster with single and double excitations (CCSD). The calculations employ the correlation-consistent basis sets, aug-cc-pV $\zeta \mathrm{Z}$ with $\zeta=\mathrm{D}, \mathrm{T}, \mathrm{Q}$. An inverse-power extrapolation formula is then utilized to obtain OR values at the complete basis set (CBS) limit. We investigate the basis set convergence for these methods and three choices of gauge: length gauge (with gauge-including atomic orbitals, LG(GIAOs), for DFT), the origin-invariant length gauge [LG(OI)], and the modified velocity gauge (MVG). The results show that all methods converge smoothly to the CBS limit and that the LG(OI) approach has a slightly faster convergence rate than the other choices of gauge. While the DFT methods reach gauge invariance at the CBS limit, CCSD does not. The significant difference between the MVG and LG(OI) results at the CBS limit, 26\%, indicates that CCSD is not quite at convergence in the description of electron correlation for this property. On the other hand, gauge invariance at the CBS limit for DFT does not lead to the same OR values for the two density functionals, which is also due to electron correlation incompleteness. A limited comparison to gas-phase experimental OR values for the DFT methods shows that CAM-B3LYP seems more accurate than B3LYP. Overall, this study shows that the LG(OI) approach with the aug-cc-pVTZ basis set for DFT, and with the CBS(DT) extrapolation for CCSD, provides a good cost/accuracy balance.
\end{abstract}

\section{Introduction}

Optical rotation (OR) refers to the phenomenon of rotation of the plane of polarization of impinging linearly polarized light by an optically active sample, e.g., a sample with an enantiomeric excess of a chiral compound. ${ }^{1}$ The ubiquity of enantiospecific chiral molecules in biological systems makes the ability to differentiate enantiomers especially relevant. While experimental measurements can provide the sign and magnitude of the rotation, theoretical simulations are necessary to correlate the sign of the rotation to the structure of the molecule. Therefore, the assignment of the absolute configuration of a chiral sample requires a synergistic effort of experiment and theory.

Reliable quantum mechanical (QM) methods based on density functional theory (DFT) and coupled cluster (CC) theory have been developed for the calculation of chiroptical properties, ${ }^{2-17}$ using response theory to evaluate the appropriate OR tensor. ${ }^{12,16-21}$ However, these techniques are based on approximate solutions of the Schrödinger equation, which affect the accuracy of the calculations and may compli- 
cate the comparison with experiment. The need to effectively represent electron correlation in the calculation of OR has been well established. ${ }^{10}$ While DFT methods present a cost efficient means of accomplishing this, ${ }^{6,17,20} \mathrm{CC}$ methods maintain the allure of being systematically improvable by including higher order excitations. ${ }^{12}$

The basis set dependence of OR calculations has been investigated at DFT level. ${ }^{22-24}$ Mach and Crawford examined the basis set dependence of $\mathrm{CC}$ with single and double excitations (CCSD) with the LPol basis sets along with the augmented correlation consistent basis sets of Dunning. ${ }^{25}$ Srebro et al. also tested the LPol basis sets, showing that they do not outperform aug-cc-pVDZ. ${ }^{26}$ This work also provides insight on the potential benefits of using a functional with a long-range correction. Howard et al. investigated the performance of the ORP basis set with CC methods, ${ }^{27}$ and Haghdani et al. studied the basis set dependence of CC methods in comparison with DFT for a set of seven molecules. ${ }^{28}$

Much work in pursuit of improving accuracy and reducing computational cost of OR calculations has been done through manipulation of the basis set. The work of Cheeseman et al. has demonstrated the importance of diffuse functions for OR calculations. ${ }^{3}$ Basis sets specifically for determination of optical properties have been developed. The LPol basis sets of Baranowska and Sadlej ${ }^{29}$ augment the sets of Duijneveldt ${ }^{30}$ with diffuse and polarization functions. The ORP basis set of Baranowska and Laczkowska was later developed, ${ }^{31}$ in which the basis sets of Schäfer et al. ${ }^{32}$ were modified with contracted diffuse and polarization functions, resulting in smaller basis sets than the LPol sets. Recently our group proposed two minimal basis sets, augD-3-21G and augT3-3$21 \mathrm{G}$, both of which are formed from augmenting the $3-21 \mathrm{G}$ set with diffuse functions from the parent Dunning correlation consistent basis sets optimized for OR calculations, ${ }^{33}$ based on an initial study of Wiberg et al. with unoptimized functions. ${ }^{34}$ These basis sets were proposed with the intent of maintaining the quality of a large basis set while decreasing the cost of
OR calculations.

Another important issue of approximate calculations is that the choice of gauge for the representation of the electric dipole perturbation leads to numerically different results, contrary to a hypothetical exact calculation. ${ }^{12,14}$ Furthermore, in the length gauge (LG) the OR is origin dependent while in the velocity gauge (VG) the OR has a spurious static limit. These are both unphysical artifacts due to the incompleteness of the basis set and of the electron correlation treatment in practical simulations. The origin dependence of LG calculations can be removed in variational methods using London orbitals, also known as gauge including atomic orbitals (GIAOs), at the cost of considerably more complicated equations and computer codes. ${ }^{35,36}$ Additionally, this approach cannot be used with standard CC methods, unless one employs uncommon orbital optimization techniques. ${ }^{37,38}$ Recently, our group has introduced an approach to obtain origin invariant LG results, LG(OI), without the need of GIAOs so that it can be equally used with variational and non-variational methods. ${ }^{39,40}$ To obtain meaningful results with VG, one needs to calculate the spurious static term explicitly and subtract it out, an approach called modified VG (MVG). ${ }^{14}$

Despite all of the work mentioned above, it is not yet clear what choice of gauge, level of theory, and basis set is optimal for OR calculations. The main obstacle to such a determination has been the inability to perform a uniform comparison between choices of gauge and basis sets across variational and non-variational methods due to the unresolved issue of the origin dependence of standard LG calculations (before the development of the LG(OI) approach). Another problem is that a direct comparison with experimental data is difficult for this sensitive molecular property. On the one hand, simulations are not yet able to reliably account for solvation effects, ${ }^{41-50}$ vibrational effects are typically introduced only through approximate perturbative treatments, ${ }^{51-56}$ and proper averaging of multiple conformers of the same enantiomer with opposite OR sign requires very accurate relative energy calculations. ${ }^{57,58}$ On the other 
hand, experimental measurements of rigid chiral molecules in the gas phase (which would sidestep some of the above simulation limitations, i.e., conformational averaging and solvation effects) are very limited due to the low volatility of these compounds and the overall small signal. ${ }^{34,59-62}$

In this work, we address a number of these issues by performing a thorough analysis of the basis set dependence in approximate calculations of optical rotation using different choices of gauge. We use an extensive set of chiral molecules (50 at DFT level and 17 at CCSD level) to test how OR calculations approach the complete basis set (CBS) limit with the LG (including GIAOs for DFT), LG(OI), and MVG schemes. The calculations are performed with the aug-cc-pV $\zeta \mathrm{Z}$ series up to $\zeta=\mathrm{Q}$ followed by an extrapolation to the CBS limit, which provide a consistent improvement of the basis set description. We investigate the convergence towards the CBS limit for each choice of gauge within each method, the convergence towards gauge invariance for each method, and a comparison between methods at the CBS limit. Thus, we can draw important conclusions on the effect of basis set and electron correlation incompleteness for a given choice of gauge and model chemistry. We also attempt a limited comparison with experimental data for a subset of 5 rigid molecules for which experimental data in the gas phase are available.

The paper is organized as follows. The methods used and the strategy to analyze the data are described in Section 3, the results of the calculations are reported in Section 4, and the interpretation of the results and concluding remarks are presented in Section 5.

\section{Theory}

Although this work is based on well-established methodologies, ${ }^{12,14,16,18,39,63}$ it is useful to briefly review the main equations to compute the optical rotation. The isotropic OR is expressed as specific rotation $[\alpha]_{\omega}\left(\right.$ or $\left.[\alpha]_{\lambda}\right)$ in customary units of $\operatorname{deg}[\mathrm{dm}(\mathrm{g} / \mathrm{mL})]^{-1}$, where $\omega$ is the frequency of the impinging light (and $\lambda$ is the corresponding wavelength):

$$
[\alpha]_{\omega}=\frac{\left(72 \times 10^{-6}\right) \hbar^{2} N_{A} \omega^{2}}{c^{2} m_{e}^{2} M} \frac{\operatorname{Tr}(\boldsymbol{\beta})}{3}
$$

where $\hbar$ is the reduced Planck's constant $(\mathrm{J} \mathrm{s})$, $N_{A}$ is Avogadro's number, $c$ is the speed of light $(\mathrm{m} / \mathrm{s}), m_{e}$ is the mass of an electron $(\mathrm{Kg}), M$ is the molecular mass (amu). $\boldsymbol{\beta}$ is the electric dipole-magnetic dipole polarizability tensor, ${ }^{12}$ which is expressed in atomic units together with the frequency $\omega$. The tensor can be written with a sum-over-states (SOS) formula as:

$$
\beta_{\alpha \beta}=2 \sum_{j \neq 0} \frac{\operatorname{Im}\left\langle\psi_{0}\left|\mu_{\alpha}\right| \psi_{j}\right\rangle\left\langle\psi_{j}\left|m_{\beta}\right| \psi_{0}\right\rangle}{\omega_{j 0}^{2}-\omega^{2}}
$$

where $\psi_{0}$ and $\psi_{j}$ are the ground and $j$ th excited state wavefunctions, respectively, $\boldsymbol{\mu}$ is the electric dipole operator, $\mathbf{m}$ is the magnetic dipole operator, Greek indices correspond to Cartesian components, and $\omega_{j 0}$ is the $j$ th excitation frequency. This expression works in the non-resonant regime ${ }^{1,64-66}$ and for wavelengths larger than the average molecular size. ${ }^{67}$ In practice, the tensor is evaluated using the linear response formalism (LR), because it does not require the evaluation of excited states. ${ }^{12,14,16,18,63}$ The linear response function, expressed with the notation $\left\langle\langle X ; Y\rangle_{\omega}\right.$ for two generic operators $X$ and $Y$, can be evaluated using two representations of the electric dipole operator:

$$
\begin{aligned}
& \mu_{\alpha}^{L}=-\sum_{i} r_{i ; \alpha} \\
& \mu_{\alpha}^{V}=-\sum_{i} p_{i ; \alpha}
\end{aligned}
$$

where the summation is over the number of electrons and $\mathbf{r}$ and $\mathbf{p}$ are the position and momentum operators. The two representations are denoted length gauge ( $L$ in Eq. 3) and velocity gauge ( $V$ in Eq. 4). The trace of the tensors in the two choices of gauge is equivalent in exact calculations: ${ }^{14}$

$$
\operatorname{Im}\left\{\operatorname{Tr}\left(\left\langle\left\langle\vec{\mu}^{L} ; \vec{m}\right\rangle_{\omega}\right)\right\}=\omega^{-1} \operatorname{Re}\left\{\operatorname{Tr}\left(\left\langle\vec{\mu}^{V} ; \vec{m}\right\rangle_{\omega}\right)\right\} .\right.
$$

In the context of this work, we use gauge invariance to indicate when the different choices of representation of the dipole operator in Eqs. 
3-4 lead to the same OR value, i.e., gauge invariance refers to the equality in Eq. 5. This equivalence is also satisfied for approximate methods with variationally optimized orbitals in the CBS limit. ${ }^{14,37}$ However, achieving gauge invariance in approximate variational methods does not guarantee that the OR is numerically the same as that obtained in an exact calculation. For non-variational methods, like in standard approximations of CC theory, and in general with incomplete basis sets, one obtains numerically distinct values of the OR in Eq. 1 with different choices of gauge.

As discussed in the Introduction, approximate calculations with incomplete basis sets lead to an unphysical static limit in the velocity gauge formulation, which is explicitly calculated and subtracted out in the MVG method, although the results are intrinsically origin invariant. ${ }^{14}$ Conversely, approximate LG calculations lead to origin-dependent OR values; these can be made origin-independent using GIAOs with variational methods. ${ }^{16,35,36}$ However, GIAOs are not useful with methods where the orbital response is not included in the LR calculation, as for standard CC methods. ${ }^{18}$ Alternatively, origin independence can be achieved with the LG(OI) approach, which works for variational and non-variational methods. ${ }^{39,40}$ The origin dependence of the $\boldsymbol{\beta}$ tensor in the LG is due to the off-diagonal elements of the mixed length-velocity gauge electric dipole-electric dipole polarizability tensor $\boldsymbol{\alpha}^{(L, V)}$, which is non-symmetric for approximate methods. ${ }^{39,40,68,69}$ In LG(OI), $\boldsymbol{\alpha}^{(L, V)}$ is diagonalized with a singular value decomposition (SVD):

$$
\boldsymbol{\alpha}^{(L, V)}=\boldsymbol{U} \boldsymbol{\alpha}_{\boldsymbol{D}} \boldsymbol{V}^{\dagger}
$$

and the inverse transformation is applied to the $\boldsymbol{\beta}$ tensor:

$$
\tilde{\boldsymbol{\beta}}=\boldsymbol{U}^{\dagger} \boldsymbol{\beta} \boldsymbol{V}
$$

This transformation renders the diagonal elements of $\tilde{\boldsymbol{\beta}}$, and therefore its trace, origin invariant. Since the $\boldsymbol{\alpha}^{(L, V)}$ tensor is symmetric in an exact calculation, the LG, LG(OI), and MVG methods become equivalent for a complete treatment of electron correlation and a complete basis set. ${ }^{40}$ For variational methods, the choice of gauge [LG(GIAOs), LG(OI), or MVG] becomes immaterial in the CBS limit even with approximate treatments of electron correlation, as demonstrated numerically in section 4 .

\section{Methods}

The OR was computed using standard linear response methods ${ }^{18,63}$ as discussed in section 2. Three choices of gauge were considered: modified velocity gauge (MVG), ${ }^{14}$ length gauge (LG), as well as the newly developed origininvariant length gauge [LG(OI)]. ${ }^{39,40}$ LG calculations were performed with gauge-including atomic orbitals (GIAOs) ${ }^{35,36}$ for variational methods (B3LYP, ${ }^{70}$ CAM-B3LYP ${ }^{71}$ ) and without for CCSD. Thus, CCSD-LG OR calculations are inherently origin dependent. The two density functionals were chosen as representative members that provide reasonable OR values. ${ }^{26}$

A test set of 50 molecules with a wide range of molecular sizes and OR magnitudes was compiled from previous OR studies, ${ }^{25-27,72}$ see Figure 1. Molecular geometries were also obtained from these sources, as detailed in Tables S1-S50 in the Supporting Information (SI). OR values were calculated for the whole set using DFT methods and for the first 17 molecules in Figure 1 using CCSD because of computational limitations.

Basis sets of various sizes were used in order to determine the tendency of OR values to converge for a given method/gauge. Since it is important to obtain a consistent trend towards the basis set limit, ${ }^{40}$ the correlation consistent basis set series was used: aug-cc-pV $\zeta \mathrm{Z}$, where $\zeta$ $=\mathrm{D}, \mathrm{T}$, or $\mathrm{Q}$. OR values were extrapolated to the CBS limit using the two point inverse power extrapolation formula: ${ }^{73}$

$$
[\alpha]_{\lambda}^{\infty}=[\alpha]_{\lambda}^{\zeta}-A \zeta^{-n}
$$

where $[\alpha]_{\lambda}^{\zeta}$ is the OR value obtained for the 


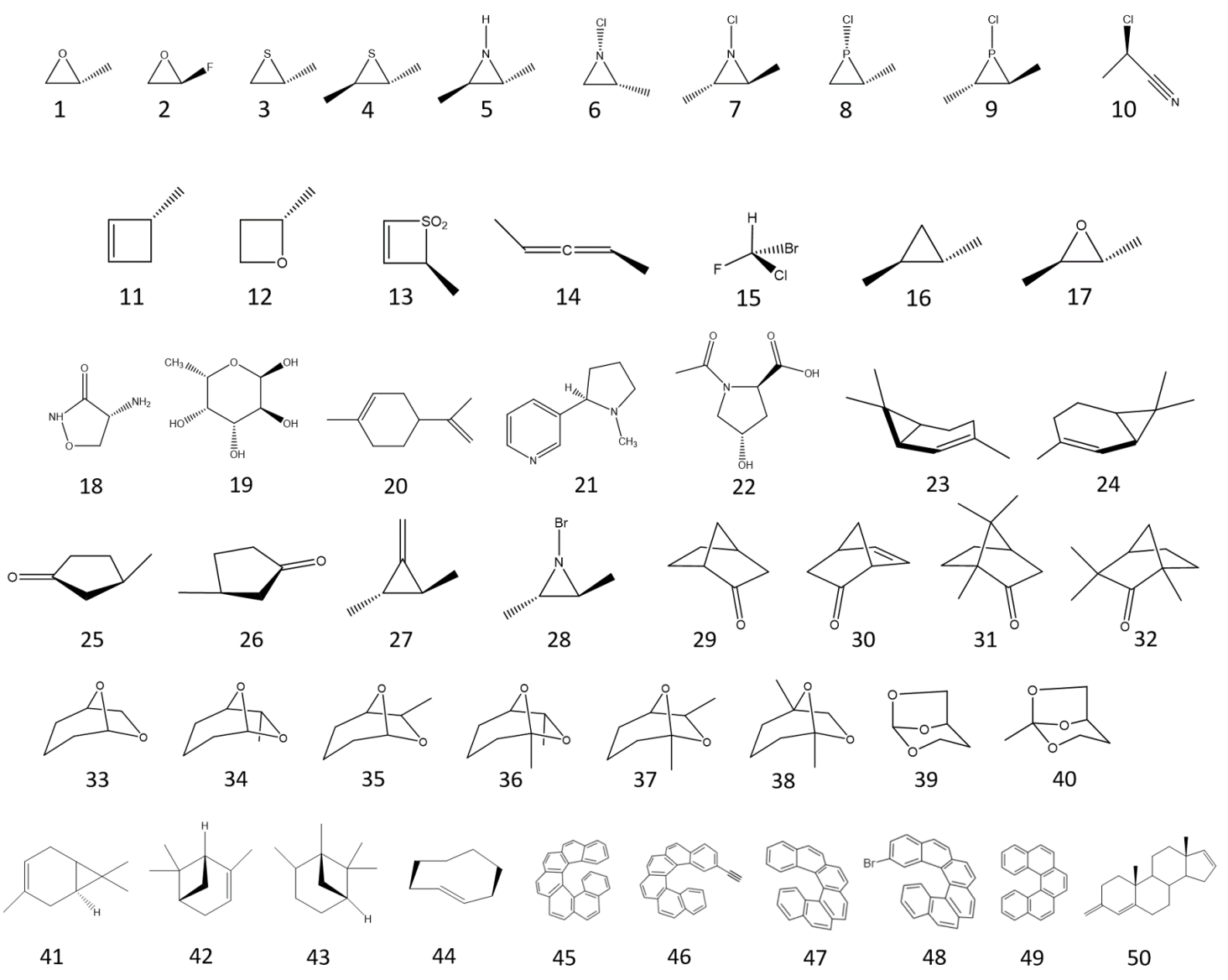

Figure 1: The test set of molecules used for OR calculations is shown. Molecules 1-17 were used with both CCSD and DFT methods, whereas 18-50 were used exclusively with the DFT methods. ${ }^{25-27,72}$

basis set aug-cc-pV $\zeta \mathrm{Z}, A$ and $n$ are fitting parameters, and $[\alpha]_{\lambda}^{\infty}$ gives the OR value extrapolated to the CBS limit. In order to obtain a two point fitting for this system of equations, $n$ must be fixed. Values of $n$ were set to 3 for double/triple (DT) extrapolations and 5 for triple/quadruple (TQ) extrapolations, following the recommendation of Haghdani et al. ${ }^{28}$ The CBS values from the TQ extrapolations are utilized as the reference values in the comparisons, as described below. Basis sets with higher order angular momentum functions would provide results closer to the basis set limit, but we found that quadruple- $\zeta$ level was sufficient for the purposes of this study and it allows calculations on fairly large systems in a reasonable amount of time.

The OR values are analyzed to compare convergence towards the CBS limit within each choice of gauge, between gauges within each method, and between methods. The comparison is performed using the signed relative error for each molecule $i$ at a given basis set size, normalized by the total number of molecules in the set $(N)$ :

$$
\Delta_{i}^{\mathrm{ref}}=\frac{1}{N} \frac{[\alpha]_{i}-[\alpha]_{i}^{\mathrm{ref}}}{[\alpha]_{i}^{\mathrm{ref}}}
$$

By changing the OR value used as reference, one can focus on a different type of comparison. For instance, by using the (best estimate) CBS OR value within each gauge for a given method as reference, once can probe the basis set convergence of each choice of gauge. If the CBS OR value for a specific choice of gauge is used as reference, one can test gauge invariance for a given method as a function of the basis set size. Finally, by using the CBS OR value for a specific choice of gauge and method, one 
can compare differences between methods. For each molecule in the test set, we selected the enantiomer with positive OR (when calculated with the largest basis set) to avoid complications in the interpretation of the sign of $\Delta_{i}^{\text {ref }}$ in Eq. 9 ; thus, $\Delta_{i}^{\text {ref }}<0$ always indicates an underestimation of the reference value and vice versa. The molecules for which we changed enantiomer compared to the original study are also listed in the SI. The OR signs are consistent for all molecules and basis sets, with a few exceptions with aug-cc-pVDZ that are explicitly discussed in the next section.

The $\Delta_{i}^{\text {ref }}$ values for each molecule are displayed using stacked bar graphs. Since each $\Delta_{i}^{\text {ref }}$ is normalized for the number of molecules used with a particular level of theory, the entire length of each column in the graphs gives the mean unsigned relative error (MURE):

$$
\Delta_{U}^{\mathrm{ref}}=\sum_{i=1}^{N}\left|\Delta_{i}^{r e f}\right|
$$

Horizontal bars crossing through each column at the midpoint correspond to half the value of the mean signed relative error (MSRE):

$$
\Delta_{S}^{\mathrm{ref}}=\sum_{i=1}^{N} \Delta_{i}^{r e f}
$$

The magnitude of the OR across the test set varies between $O(1)-O\left(10^{3}\right)$ $\operatorname{deg}[\mathrm{dm}(\mathrm{g} / \mathrm{mL})]^{-1}$. In the plots, each $\Delta_{i}^{\text {ref }}$ bar is color coded from blue to red with increasing magnitude of the reference OR value for each molecule. The change in color was restricted to a range of 0 to $100 \mathrm{deg}[\mathrm{dm}(\mathrm{g} / \mathrm{mL})]^{-1}$, such that the bars for molecules with OR values exceeding $100 \mathrm{deg}[\mathrm{dm}(\mathrm{g} / \mathrm{mL})]^{-1}$ are displayed as the same color (red). This is done for ease of comparison as a few of the molecules, in particular the helicenes (45-49 in Figure 1), exhibit very large OR values relative to the rest of the set. This approach is slightly altered in the experimental comparisons, where the maximal value for color coding is set to $500 \mathrm{deg}[\mathrm{dm}(\mathrm{g} / \mathrm{mL})]^{-1}$ to more easily distinguish the molecules in the given set. This color coding visually provides information about any possible correlation between the sign of the error and the magnitude of the OR. Note that, although relative errors tend to be larger for molecules with small-magnitude OR, this is not a major issue for the data and it becomes less significant as the basis set size increases. On the other hand, using a single definition of the error as in Eq. 9 (plus the color coding) allows us to treat the entire test set on the same footing. It also allows us to combine information about the distribution of errors into a single plot.

Calculations used for theoretical comparisons were performed at the sodium D line $(589.3 \mathrm{~nm})$ to avoid any potential issue with resonance conditions, whereas those used for the comparison with experiment were performed at $355 \mathrm{~nm}$ and $633 \mathrm{~nm}$ to match the available experimental values. All calculations were performed using a development version of the GAUSSIAN ${ }^{74}$ suite of programs and all raw data is reported in Tables S1-S50 of the SI.

\section{Results}

The data in Figure 2 describe the degree to which the OR values converge with increasing basis set size for each choice of gauge and method. In these plots, the reference value used in Eq. 9 is the CBS(TQ) extrapolation for each gauge. For all methods, there is a clear decrease in total error compared to the reference values; the $\Delta_{U}^{\mathrm{TQ}}$ values (Eq. 10) with aug-ccpVQZ are of the order of 0.01 for the DFT methods, panels (a) and (b), and 0.02-0.04 for CCSD, panel (c). Nonetheless, MVG results show a slightly slower convergence compared to the other choices of gauge.

With smaller basis sets, especially $\zeta=\mathrm{D}$, the error is driven by the molecules with smallmagnitude OR as expected. In particular, the molecules that have the wrong sign with this basis set (molecule 27 for DFT and 2 for CCSD) account for a large part of the error, e.g., $18 \%$ of the total $\Delta_{U}^{\mathrm{TQ}}$ with aug-cc-pVDZ and LG(OI) for B3LYP and 44\% with CAM-B3LYP. Significant improvement is observed going from $\zeta=$ $\mathrm{D}$ to $\mathrm{T}$, with $\Delta_{U}^{\mathrm{TQ}}$ values going from $0.2-0.3$ 
to 0.04-0.08 for DFT, respectively. However, CBS(DT) extrapolations seem to offer little improvement and even slightly worsen the result in the case of LG.

(a)

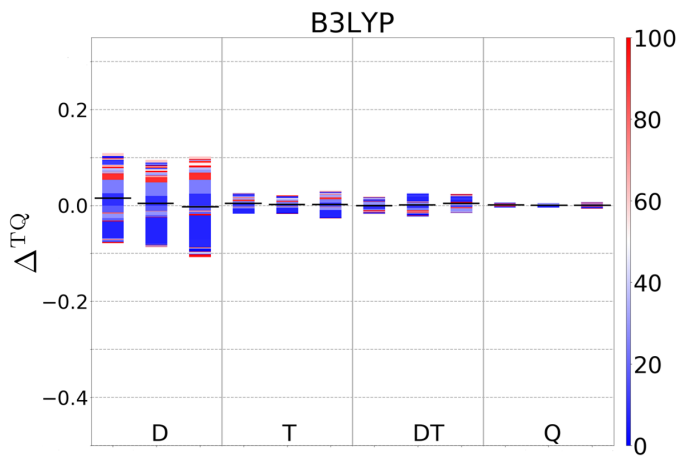

(b)

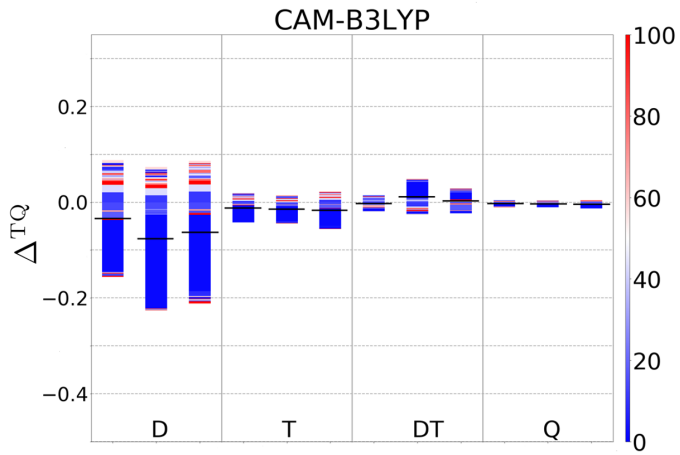

(c)

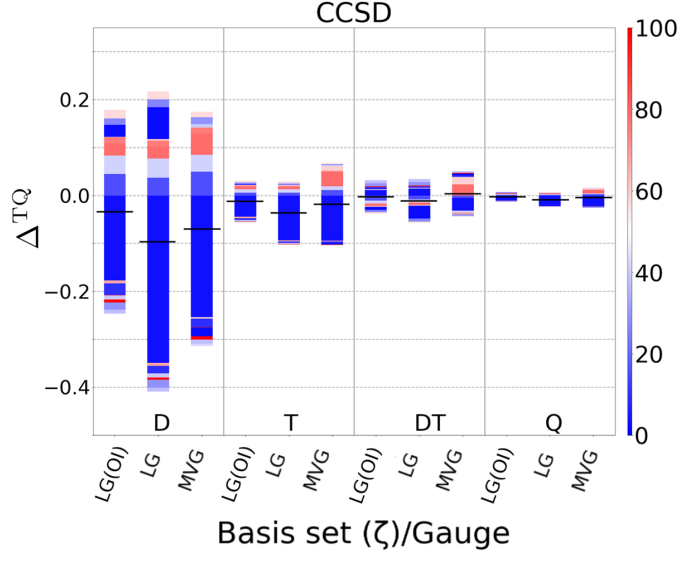

Figure 2: $\Delta^{\mathrm{TQ}}$ values for (a) B3LYP, (b) CAMB3LYP, and (c) CCSD. The reference value used for each is from the TQ extrapolation and within its own method/gauge. The color scale represents the magnitude of the reference OR value for a given molecule, where all molecules whose OR exceeds $100 \mathrm{deg}[\mathrm{dm}(\mathrm{g} / \mathrm{mL})]^{-1}$ are assigned the same maximal color value. The horizontal black lines correspond to the center point of each bar, i.e. $0.5 \Delta_{S}^{\mathrm{TQ}}$ as in Eq. 11 .

Comparing the convergence across methods, B3LYP shows the fastest convergence with ba- sis set size, but CAM-B3LYP is only slightly worse. Not surprisingly, CCSD shows a more marked basis set dependence than DFT. Nevertheless, $\Delta_{U}^{\mathrm{TQ}}$ values with aug-cc-pVQZ only vary between 0.02 for LG(OI) to 0.04 for MVG. $\Delta_{U}^{\mathrm{TQ}}$ is always largest with MVG at CCSD level compared to the other choices of gauge; in fact, the MVG error is twice as large as that of $\mathrm{LG}(\mathrm{OI})$ for basis sets with $\zeta=\mathrm{T}$ and $\mathrm{Q}$. The data in Figure 2 also show a rather random distribution of the errors and no particular correlation with the OR magnitude. The $\Delta_{S}^{\mathrm{TQ}}$ values (black horizontal lines crossing the bars in the plots) tend to be negative, indicating an overall underestimation of the reference values. However, this is again mostly driven by the molecules with small OR magnitude, especially those with the wrong sign from the $\zeta=\mathrm{D}$ calculations.

Gauge invariance is tested by selecting the CBS(TQ) extrapolation with LG(OI) as reference for all choices of gauge within a method, and the results are shown in Figure 3. This choice of reference is somewhat arbitrary, in the sense that any of the gauges could be used. However, LG(OI) was selected because of its slightly faster convergence rate towards the CBS limit compared to the other choices of gauge. Since gauge invariance is expected for variational methods at the CBS limit, it is worth discussing the DFT and CCSD results separately. For the functionals in panels (a) and (b), gauge invariance is indeed achieved at the $\mathrm{CBS}(\mathrm{TQ})$ values $\left(\Delta_{U}^{\mathrm{TQ} / \mathrm{LG}(\mathrm{OI})}=0.01\right.$ across the board), and it is practically obtained already with the aug-cc-pVQZ basis set. Differences between gauges are already small with the $\zeta=T$ basis set $\left(\Delta_{U}^{\mathrm{TQ} / \mathrm{LG}(\mathrm{OI})}<0.1\right)$, but also in this case the CBS(DT) extrapolation does not provide a significant improvement (in fact, it increases the $\Delta_{U}^{\mathrm{TQ} / \mathrm{LG}(\mathrm{OI})}$ value for LG(GIAO)). The error sign is again not related to the OR magnitude, and the $\Delta_{S}^{\mathrm{TQ} / \mathrm{LG}(\mathrm{OI})}$ values are fairly close to 0 ; the only exception is the effect of molecule 27 with the smallest basis set, see panel (b) for CAM-B3LYP.

It is apparent from Figure 3(c) that CCSD does not approach gauge invariance at the CBS 
limit (the results from Figure 2 indicate that the CBS(TQ) extrapolation is sufficiently close to the CBS limit). Gauge invariance is not expected for standard approximate $\mathrm{CC}$ methods unless the treatment of electron correlation is complete, as discussed in section 2 and demonstrated numerically in Ref. 40. Therefore, the difference between choices of gauge at the CBS limit is a measure of the electron correlation incompleteness for a particular level of truncation in the excitation expansion. A value of $\Delta_{U}^{\mathrm{TQ} / \mathrm{LG}(\mathrm{OI})}=0.26$ for the $\mathrm{CBS}(\mathrm{TQ})$ extrapolation with the MVG indicates that CCSD is in fact still quite far from convergence in the treatment of electron correlation (at least for this test set). The $\Delta_{U}^{\mathrm{TQ} / \mathrm{LG}(\mathrm{OI})}$ change for $\mathrm{LG}$ by a factor of 3 (from 0.44 with aug-cc-pVDZ to 0.13 at the CBS limit) indicates a considerable decrease in the "origin dependence" for this choice of gauge. In comparison, the corresponding change for MVG is only by a factor of 2 (from 0.59 to 0.26 ). It can also be inferred from the graph that the MVG tends to give OR values that are larger in magnitude relative to $\mathrm{LG}(\mathrm{OI})$, as evidenced by the positive values of $\Delta_{S}^{\mathrm{TQ} / \mathrm{LG}(\mathrm{OI})}$ from MVG across all basis set sizes. The opposite can be said for LG, which shows a tendency to underestimate the $\mathrm{LG}(\mathrm{OI})$ reference OR.

The final comparison is across methods, which can be obtained by using the CBS(TQ) extrapolation with CAM-B3LYP for each gauge as reference for the other methods and basis sets (note that here there is no direct comparison across choices of gauge), see Figure 4. The results in panel (a) between different functionals indicate that gauge invariance does not lead to the same overall OR values, as the $\Delta_{U}^{\mathrm{TQ} / \mathrm{CAM}-\mathrm{B} 3 \mathrm{LYP}}$ converge to 0.26 at the CBS limit. A reduction in $\Delta_{U}^{\mathrm{TQ} / \mathrm{CAM}-\mathrm{B} 3 \mathrm{LYP}}$ can be seen from the double to triple zeta basis set. However, further increase in basis set size yields little to no improvement. This is due to results from both functionals converging at similar rates and in the same direction to their respective CBS values, such that the difference between OR values remains almost constant as the basis set size is increased. Posi- (a)

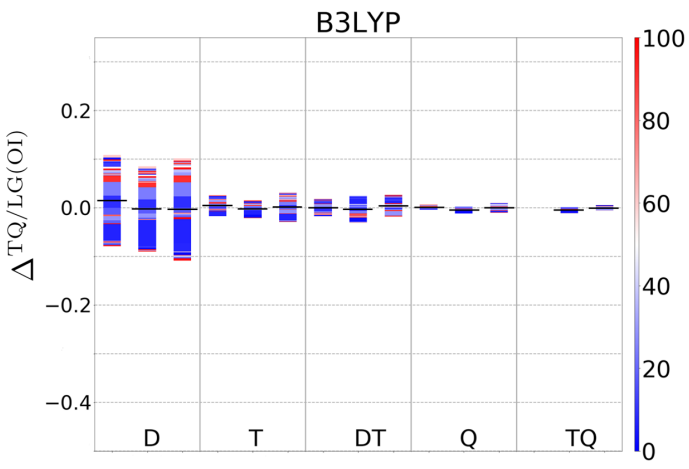

(b)

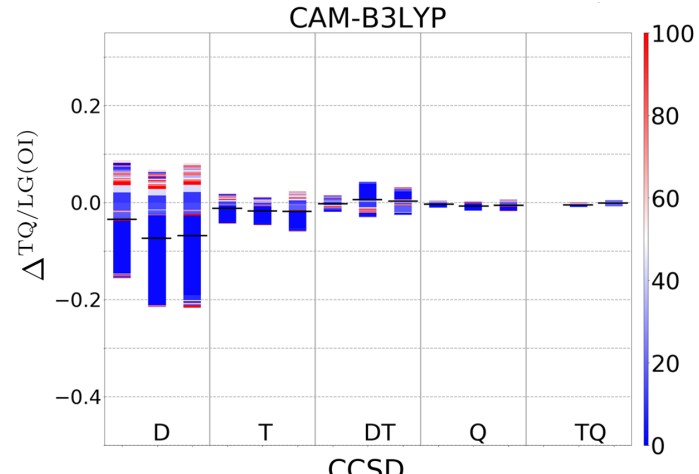

(c)

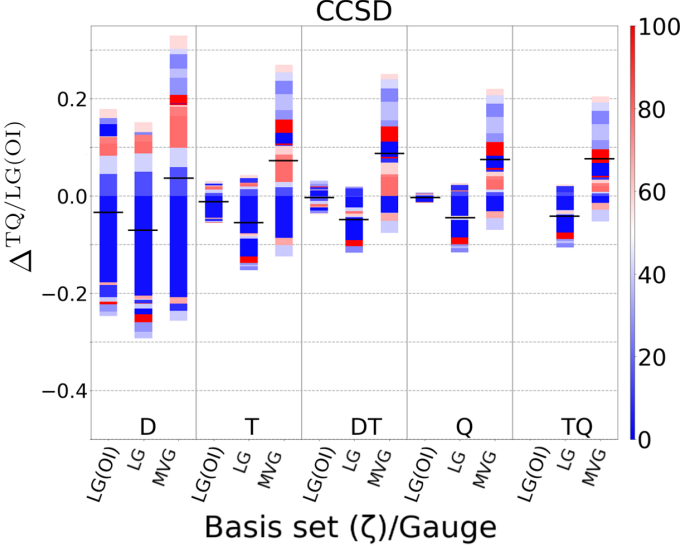

Figure 3: $\Delta^{\mathrm{TQ} / \mathrm{LG}(\mathrm{OI})}$ values for (a) B3LYP, (b) CAM-B3LYP, and (c) CCSD. The reference value used for each is from the TQ extrapolation using $\mathrm{LG}(\mathrm{OI})$. The color scale represents the magnitude of the reference OR value for a given molecule, where all molecules whose OR exceeds $100 \mathrm{deg}[\mathrm{dm}(\mathrm{g} / \mathrm{mL})]^{-1}$ are assigned the same maximal color value. The horizontal black lines correspond to the center point of each bar, i.e. $0.5 \Delta_{S}^{\mathrm{TQ}}$ as in Eq. 11 . 
tive $\Delta_{S}^{\mathrm{TQ} / \mathrm{CAM}-\mathrm{B} 3 \mathrm{LYP}}$ values indicate that B3LYP tends to provide larger OR values than CAMB3LYP overall. However, while $\zeta=\mathrm{D}$ B3LYP tends to provide larger $\mathrm{OR}$ values for molecules with large-magnitude OR, the distribution of values becomes more homogeneous with larger basis sets.
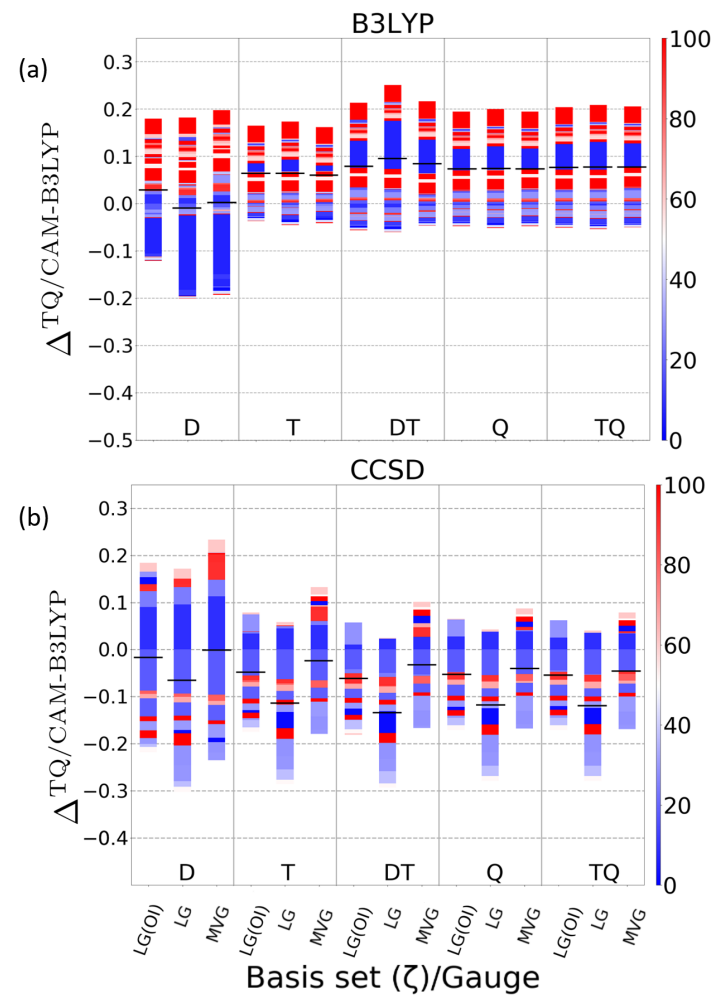

Figure 4: $\Delta^{\text {TQ/CAM-B3LYP }}$ for (a) B3LYP and (b) CCSD using the CAM-B3LYP TQ extrapolation as reference; each gauge is compared with itself. The color scale represents the magnitude of the reference OR value for a given molecule, where all molecules whose OR exceeds $100 \mathrm{deg}[\mathrm{dm}(\mathrm{g} / \mathrm{mL})]^{-1}$ are assigned the same maximal color value. The horizontal black lines correspond to the center point of each bar, i.e. $0.5 \Delta_{S}^{\mathrm{TQ}}$ as in Eq. 11 .

The comparison with CCSD in Figure 4(b) (on only the 17 molecules used for this level of theory) shows some similarities with the previous data set. At the CBS limit, $\Delta_{U}^{\mathrm{TQ} / \mathrm{CAM} \text {-B3LYP }}$ is 0.23 for $\mathrm{LG}(\mathrm{OI})$ and 0.25 for $\mathrm{MVG}$, after steadily decreasing from the $\zeta=\mathrm{D}$ values. However, the position of the error bars is different for different choices of gauge (see the black lines corresponding to $\Delta_{S}^{\mathrm{TQ} / \mathrm{CAM}-\mathrm{B} 3 \mathrm{LYP}}$ in the plot) due to the lack of gauge invariance for CCSD. The negative $\Delta_{S}^{\mathrm{TQ} / \mathrm{CAM}-\mathrm{B} 3 \mathrm{LYP}}$ values indicate that CCSD provides consistently smaller OR values than CAM-B3LYP.

A comparison with experimental data (used as reference in Eq. 10) is performed for a subset of the molecules: 14,30 , and 41-43, shown in Figure 5. These compounds were chosen because they are fairly rigid and have a single stable conformer, and experimental data in the gas phase are available. ${ }^{34,62}$ This choice eliminates various difficulties due to solvation effects and averaging over contributions from multiple stable conformers, but it comes at the cost of a limited sample size. Furthermore, contributions from vibrational motions are not included in the calculations. Therefore, these constraints somewhat limit the generality of this comparison. Only DFT methods are used because most of these systems are too large to be treated at CCSD level with large basis sets.

At $355 \mathrm{~nm}$, left panels in Figure 5, the errors are dominated by molecule 30 , which is a notoriously difficult case for simulations. ${ }^{62,75}$ The increase in basis set size does not provide a significant improvement of the results. More informative are the results at $633 \mathrm{~nm}$, shown in the right panels in Figure 5, where the errors are more evenly distributed. Also in this case, the basis set incompleteness is not the most important source of error; this is the case also for the choice of gauge with smaller basis sets. Overall, CAM-B3LYP shows a smaller $\Delta_{U}^{\text {Exp }}$ with each choice of gauge and basis set compared to B3LYP by about 0.05-0.15 units. The smallest $\Delta_{U / S}^{\text {Exp }}$ values are obtained for both methods with $\mathrm{LG}(\mathrm{OI})$ and the CBS(DT) extrapolation, which is likely fortuitous. From this data, it is not possible to determine whether the disagreement with experiment at the CBS limit is due to missing vibrational effects or electron correlation incompleteness in the calculations, although it is likely that both contributions are important. 

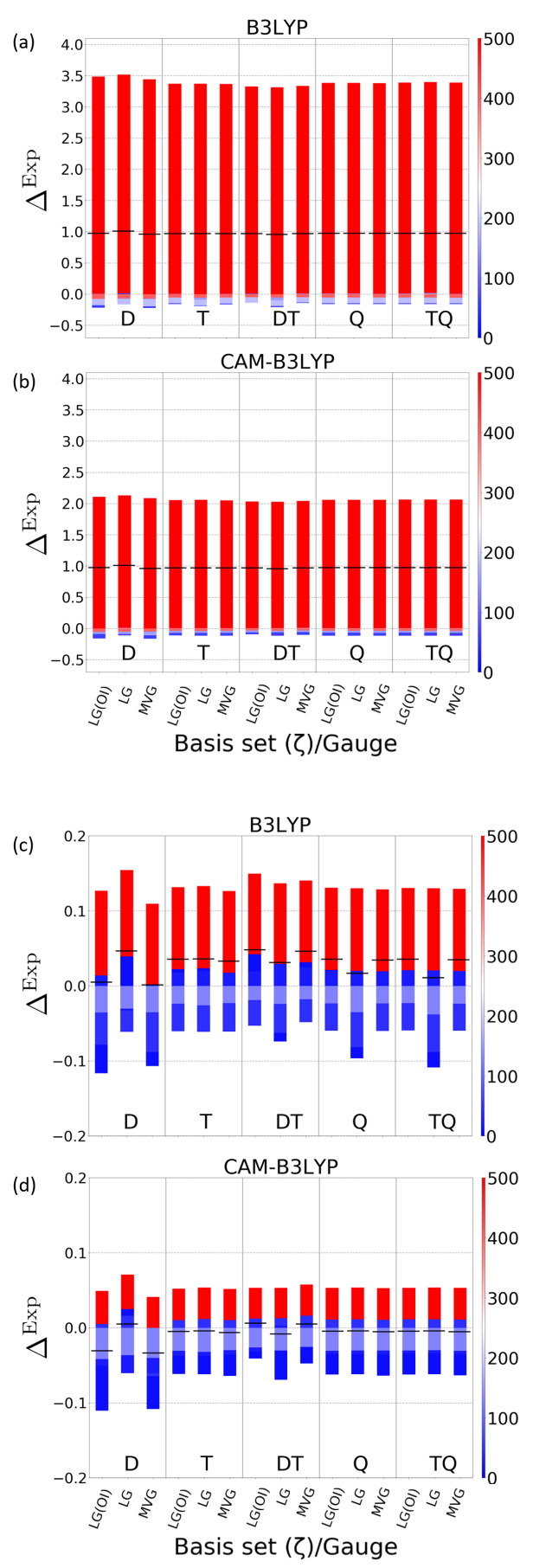

Figure 5: $\Delta^{\operatorname{Exp}}$ values are shown for B3LYP in (a) and CAM-B3LYP in (b) at $355 \mathrm{~nm}$ and for B3LYP in (c) and CAM-B3LYP in (d) at $633 \mathrm{~nm}$. Reference values are taken from gasphase experiments. Color scale represents magnitude of the reference OR value for a given molecule, where all molecules whose OR exceeds $500 \mathrm{deg}[\mathrm{dm}(\mathrm{g} / \mathrm{mL})]^{-1}$ are assigned the same maximal color value. The horizontal black lines correspond to the center point of each bar, i.e. $0.5 \Delta_{S}^{\mathrm{TQ}}$ as in Eq. 11 .

\section{Discussion and Conclu- sions}

In this work, we examine how OR calculations progress towards the CBS limit with different choices of gauge and level of theory. We use an extensive test set of 50 molecules for DFT methods and 17 molecules for CCSD, Dunning basis sets up to quadruple $\zeta$ with a two-point inverse power extrapolation to estimate the CBS limit, and three choices of gauge: MVG, LG(OI) and LG (including GIAOs for DFT).

The plots in Figures 2-3 show that the TQ extrapolation is essentially at the CBS limit for DFT, with both functionals showing gauge invariance. For CCSD, the convergence towards the CBS limit is slower than for DFT, and it is due to the larger dependence of this level of theory on the basis set size compared to DFT. The CCSD $\Delta_{U}^{\mathrm{TQ}}$ values in Figure 2, up to 0.04, are slightly larger than for DFT, but they are small enough to consider the OR values essentially converged with respect to basis set size. All methods provide an almost perfect consistency in the OR sign with all basis sets, choices of gauge, and molecules. The only exceptions are molecules 27 for DFT and 2 for CCSD, which have a small OR magnitude and the calculations with the smallest basis set give the wrong sign with all choices of gauge. These two molecules are the main cause of the large relative errors with the aug-cc-pVDZ basis set in Figure 2. The CBS(DT) extrapolation offers little to no improvement over a regular aug-ccpVTZ calculation for DFT, likely because the double $\zeta$ results are of poor quality. On the other hand, the CBS(DT) extrapolation provides significant improvement over the triple $\zeta$ results for CCSD-MVG, because this choice of gauge has a slower rate of convergence with basis set size. The slower convergence rate for MVG compared to LG and LG(OI) is consistent across methods (although the differences between gauges are small for DFT). In all cases, the results with the CBS(DT) extrapolation are farther from the CBS limit than those obtained with the aug-cc-pVQZ basis set, compare DT and $\mathrm{Q}$ sectors in the plots in Figure 2. Nev- 
ertheless, the aug-cc-pVQZ basis set is often prohibitive for production level calculations; therefore, using aug-cc-pVTZ for DFT calculations and the same basis with a CBS(DT) extrapolation for CCSD may provide the best cost/accuracy balance for OR calculations.

CCSD does not reach gauge invariance at the CBS limit, as shown in Figure 3(c), with a difference of $\Delta_{U}^{\mathrm{TQ} / \mathrm{LG}(\mathrm{OI})}$ between $\mathrm{LG}(\mathrm{OI})$ and MVG equal to 0.26 (i.e., $26 \%$ ). This indicates that the treatment of electron correlation for this property is not quite at convergence for CCSD, at least for this test set. It would be interesting to see what level of CC expansion (and basis set size) is necessary to reach gauge invariance for these molecules, but these calculations are currently beyond our capabilities. While gauge invariance for the systematically improvable CC methods would correspond to the exact $\mathrm{OR}$ values for a molecule (at least in terms of electronic response), gauge invariance provides no such guarantee for approximate density functionals. This is clearly shown in Figure 4(a), where B3LYP and CAM-B3LYP do not reach the same CBS limit even if they individually reach gauge invariance. A known limitation of current density functionals is that a systematic improvement of electron correlation for a particular functional is not possible, although Autschbach and coworkers have shown that range-separated functionals can provide better optical activity results when the rangeseparation parameter is optimized to reduce the delocalization error. ${ }^{76}$

An important question is whether these methods provide OR estimates that become closer to the experimental results as the basis set is increased. This is a difficult question to answer because it is hard to perform a clean comparison with experimental data. In fact, solvation effects, signal averaging over multiple conformers of the same enantiomer, and vibrational contributions to the OR are all factors that significantly affect the final measured value of this property. Theoretical models to account for these effects are still rather approximate and would cloud the comparison. Therefore, we only attempted a limited comparison by focusing on 5 rigid molecules with a single stable conformer for which experimental data in gas phase are available. This comparison still suffers from the lack of vibrational effects in the calculations. With these caveats in mind, the results in Figure 5 indicate that CAM-B3LYP is more accurate than B3LYP for every basis set/gauge choice and the aug-cc-pVTZ basis set is sufficient to obtain good agreement with experiment. The long-range part 77 of CAMB3LYP allows a more accurate modeling of the outermost parts of the wavefunction, which is especially important for OR calculations.

From all plots, it is evident that the choice of gauge is less important than the choice of basis set and method. However, Figure 2 shows that the $\mathrm{LG}(\mathrm{OI})$ results converge faster than MVG to the CBS limit for all methods, especially for CCSD. Considering that LG(OI) is computationally cheaper than MVG and that it is less difficult to implement than LG with GIAOs for DFT, this choice of gauge may be optimal for OR calculations.

\section{Supporting Information}

The Supporting Information includes the OR values for each of the molecules discussed in the main text (Tables S1-S50). Additionally, values for $\Delta_{\mathrm{U}}^{\mathrm{ref}}$ are shown in Tables S51-S54, and $\Delta_{\mathrm{S}}^{\text {ref }}$ values are given in Tables S55-S58.

Acknowledgement The authors gratefully acknowledge support from the National Science Foundation through Grant No. CHE-1650942.

\section{References}

(1) Barron, L. D. Molecular Light Scattering and Optical Activity, 2nd ed.; Cambridge University Press, Cambridge, 2004.

(2) Polavarapu, P. L. Ab initio molecular optical rotations and absolute configurations. Mol. Phys. 1997, 91, 551-554.

(3) Cheeseman, J. R.; Frisch, M. J.; Devlin, F. J.; Stephens, P. J. Hartree-Fock and Density Functional Theory ab Initio Calculation of Optical Rotation Using 
GIAOs: Basis Set Dependence. J. Phys. Chem. A 2000, 104, 1039-1046.

(4) Grimme, S. Calculation of frequency dependent optical rotation using density functional response theory. Chem. Phys. Lett. 2001, 339, 380-388.

(5) Polavarapu, P. L. Optical rotation: recent advances in determining the absolute configuration. Chirality 2002, 14, 768-781.

(6) Autschbach, J.; Patchkovskii, S.; Ziegler, T.; van Gisbergen, S. J. a.; Jan Baerends, E. Chiroptical properties from time-dependent density functional theory. II. Optical rotations of small to medium sized organic molecules. J. Chem. Phys. 2002, 117, 581-592.

(7) Ruud, K.; Helgaker, T. Optical rotation studied by density-functional and coupledcluster methods. Chem. Phys. Lett. 2002, 352, 533-539.

(8) Stephens, P. J.; McCann, D. M.; Cheeseman, J. R.; Frisch, M. J. Determination of absolute configurations of chiral molecules using ab initio time-dependent density functional theory calculations of optical rotation: How reliable are absolute configurations obtained for molecules with small rotations? Chirality 2005, 17, $52-64$.

(9) Grimme, S.; Bahlmann, A.; Haufe, G. Ab initio calculations for the optical rotations of conformationally flexible molecules: A case study on six-, seven-, and eightmembered fluorinated cycloalkanol esters. Chirality 2002, 14, 793-797.

(10) Ruud, K.; Stephens, P. J.; Devlin, F. J.; Taylor, P. R.; Cheeseman, J. R.; Frisch, M. J. Coupled-cluster calculations of optical rotation. Chem. Phys. Lett. 2003, 373, 606-614.

(11) Tam, M. C.; Russ, N. J.; Crawford, T. D. Coupled cluster calculations of optical rotatory dispersion of (S)-methyloxirane. J. Chem. Phys. 2004, 121, 3550-3557.
(12) Crawford, T. D. Ab initio calculation of molecular chiroptical properties. Theor. Chem. Acc. 2006, 115, 227-245.

(13) Crawford, T. D.; Stephens, P. J. Comparison of time-dependent density-functional theory and coupled cluster theory for the calculation of the optical rotations of chiral molecules. J. Phys. Chem. A. 2008, 112, 1339-1345.

(14) Pedersen, T. B.; Koch, H.; Boman, L.; Sánchez de Merás, A. M. Origin Invariant Calculation of Optical Rotation without Recourse to London Orbitals. Chem. Phys. Lett. 2004, 393, 319-326.

(15) Crawford, T. D.; Owens, L. S.; Tam, M. C.; Schreiner, P. R.; Koch, H. $\mathrm{Ab}$ initio calculation of optical rotation in (P)-(+)-[4]triangulane. J. Am. Chem. Soc. 2005, 127, 1368-1369.

(16) Krykunov, M.; Autschbach, J. Calculation of optical rotation with time-periodic magnetic-field-dependent basis functions in approximate time-dependent densityfunctional theory. J. Chem. Phys. 2005, 123, 114103.

(17) Autschbach, J. Computing chiroptical properties with first-principles theoretical methods: background and illustrative examples. Chirality 2009, 21, E116-E152.

(18) Koch, H.; Jø rgensen, P. Coupled cluster response functions. J. Chem. Phys. 1990, 93, 3333-3344.

(19) Christiansen, O.; Jorgensen, P.; Hattig, C. Response functions from Fourier component variational perturbation theory applied to a time-averaged quasienergy. Int. J. Quantum Chem. 1998, 68, 1-52.

(20) Autschbach, J. Time-dependent density functional theory for calculating originindependent optical rotation and rotatory strength tensors. ChemPhys Chem 2011, 12, 3224-3235. 
(21) Zhang, K.; Balduf, T.; Caricato, M. Full optical rotation tensor at coupled cluster with single and double excitations level in the modified velocity gauge. Chirality 2021, 33, 303-314.

(22) Campos, C.; Jorge, F.; Silva, T.; Coppo, M. Basis set convergence on optical rotation DFT calculations. Chem. Phys. Lett. 2010, 494, 170-173.

(23) Jorge, F. E.; de Oliveira, A. Z.; Silva, T. P. CAM-B3LYP optical rotations at different wavelengths: Comparison with CCSD results. Int. J. Quantum. Chem. 2016, 116, 21-26.

(24) Hedegard, E. D.; Jensen, F.; Kongsted, J. Basis Set Recommendations for DFT Calculations of Gas-Phase Optical Rotation at Different Wavelengths. J. Chem. Theory Comput. 2012, 8, 4425-4433.

(25) Mach, T. J.; Crawford, T. D. Basis Set Dependence of Coupled Cluster Optical Rotation Computations. J. Phys. Chem. A 2011, 115, 10045-10051.

(26) Srebro, M.; Govind, N.; de Jong, W. A.; Autschbach, J. Optical Rotation Calculated with Time-Dependent Density Functional Theory: The OR45 Benchmark. J. Phys. Chem. A 2011, 115, 10930-10949.

(27) Howard, J. C.; Sowndarya S. V., S.; Ansari, I. M.; Mach, T. J.; BaranowskaLaczkowska, A.; Crawford, T. D. Performance of Property-Optimized Basis Sets for Optical Rotation with Coupled Cluster Theory. J. Phys. Chem. A 2018, 122, 5962-5969.

(28) Haghdani, S.; A strand, P.-O.; Koch, H. Optical Rotation from Coupled Cluster and Density Functional Theory: The Role of Basis Set Convergence. J. Chem. Theory Comput. 2016, 12, 535-548.

(29) Baranowska, A.; Sadlej, A. J. Polarized basis sets for accurate calculations of static and dynamic electric properties of molecules. J. Comp. Chem. 2009, 552560 .

(30) Van Duijneveldt, F. Gaussian Basis Sets for the Atoms H-Ne for Use in Molecular Calculations, IBM. J. Res. Dev. 1971, 945 .

(31) Baranowska-Łacczkowska, A.; Łączkowski, K. Z.; Henriksen, C.; Fernández, B. New Basis Set for the Evaluation of Specific Rotation in Flexible Biological Molecules in Solution. J. Phys. Chem. A 2018, 122, 5477-5483.

(32) Schäfer, A.; Horn, H.; Ahlrichs, R. Fully optimized contracted Gaussian basis sets for atoms Li to Kr. J. Chem. Phys. 1992, 97, 2571-2577.

(33) Aharon, T.; Caricato, M. Compact Basis Sets for Optical Rotation Calculations. J. Chem. Theory Comput. 2020, 16, 44084415 .

(34) Wiberg, K. B.; Caricato, M.; Wang, Y.-G.; Vaccaro, P. H. Towards the Accurate and Efficient Calculation of Optical Rotatory Dispersion Using Augmented Minimal Basis Sets. Chirality 2013, 25, 606-616.

(35) London, F. Théorie quantique des courants interatomiques dans les combinaisons aromatiques. J. Phys. Radium 1937, 8, 397-409.

(36) Ditchfield, R. Self-Consistent Perturbation Theory of Diamagnetism I. A GaugeInvariant LCAO Method for N.M.R. Chemical Shifts. Mol. Phys. 1974, 27, 789-807.

(37) Pedersen, T. B.; Fernández, B.; Koch, H. Gauge invariant coupled cluster response theory using optimized nonorthogonal orbitals. J. Chem. Phys. 2001, 114, 69836993.

(38) Lindh, G. D.; MacH, T. J.; Crawford, T. D. The optimized orbital coupled cluster doubles method and optical rotation. Chem. Phys. 2012, 401, 125-129. 
(39) Caricato, M. Origin invariant optical rotation in the length dipole gauge without London atomic orbitals. J. Chem. Phys. 2020, 153, 151101.

(40) Caricato, M.; Balduf, T. Origin invariant full optical rotation tensor in the length dipole gauge without London atomic orbitals. J. Chem. Phys. 2021, 155, 024118.

(41) Mukhopadhyay, P.; Zuber, G.; Goldsmith, M.-R.; Wipf, P.; Beratan, D. N. Solvent Effect on Optical Rotation: A Case Study of Methyloxirane in Water. ChemPhysChem 2006, 7, 2483-2486.

(42) Mukhopadhyay, P.; Zuber, G.; Wipf, P.; Beratan, D. Contribution of a Solute's Chiral Solvent Imprint to Optical Rotation. Angew. Chem. Int. Ed. 2007, 46, 6450-6452.

(43) Kundrat, M. D.; Autschbach, J. Ab Initio and Density Functional Theory Modeling of the Chiroptical Response of Glycine and Alanine in Solution Using Explicit Solvation and Molecular Dynamics. J. Chem. Theory Comput. 2008, 4, 1902-1914.

(44) Kundrat, M. D.; Autschbach, J. Modeling of the Chiroptical Response of Chiral Amino Acids in Solution Using Explicit Solvation and Molecular Dynamics. J. Chem. Theory Comput. 2009, 5, 10511060 .

(45) Haghdani, S.; Hoff, B. H.; Koch, H.; A strand, P.-O. Solvent Effects on Optical Rotation: On the Balance between Hydrogen Bonding and Shifts in Dihedral Angles. J. Phys. Chem. A 2017, 121, 47654777.

(46) Lipparini, F.; Egidi, F.; Cappelli, C.; Barone, V. The Optical Rotation of Methyloxirane in Aqueous Solution: A Never Ending Story? J. Chem. Theory Comput. 2013, 9, 1880-1884.

(47) D’Cunha, R.; Crawford, T. D. Modeling Complex Solvent Effects on the Optical
Rotation of Chiral Molecules: A Combined Molecular Dynamics and Density Functional Theory Study. J. Phys. Chem. A 2021, 125, 3095-3108.

(48) Howard, J. C.; Crawford, T. D. Calculating Optical Rotatory Dispersion Spectra in Solution Using a Smooth Dielectric Model. J. Phys. Chem. A 2018, 122, 8557-8564.

(49) Aharon, T.; Lemler, P.; Vaccaro, P. H.; Caricato, M. Comparison of measured and predicted specific optical rotation in gas and solution phases: A test for the polarizable continuum model of solvation. Chirality 2018, 30, 383-395.

(50) Egidi, F.; Giovannini, T.; Del Frate, G.; Lemler, P. M.; Vaccaro, P. H.; Cappelli, C. A combined experimental and theoretical study of optical rotatory dispersion for (R)-glycidyl methyl ether in aqueous solution. Phys. Chem. Chem. Phys. 2019, 21, 3644-3655.

(51) Mort, B. C.; Autschbach, J. Magnitude of Zero-Point Vibrational Corrections to Optical Rotation in Rigid Organic Molecules: A Time-Dependent Density Functional Study. J. Phys. Chem. A 2005, 109, 86178623 .

(52) Mort, B. C.; Autschbach, J. A Pragmatic Recipe for the Treatment of Hindered Rotations in the Vibrational Averaging of Molecular Properties. ChemPhysChem 2008, 9, 159-170.

(53) Pedersen, T. B.; Kongsted, J.; Crawford, T. D.; Ruud, K. On the importance of vibrational contributions to small-angle optical rotation: Fluoro-oxirane in gas phase and solution. J. Chem. Phys. 2009, 130, 034310.

(54) Mort, B. C.; Autschbach, J. Temperature Dependence of the Optical Rotation in Six Bicyclic Organic Molecules Calculated by Vibrational Averaging. ChemPhysChem 2007, 8, 605-616. 
(55) Ruud, K.; Taylor, P. R.; Åstrand, P.-O. Zero-point vibrational effects on optical rotation. Chem. Phys. Lett. 2001, 337, 217-223.

(56) Wiberg, K. B.; Vaccaro, P. H.; Cheeseman, J. R. Conformational Effects on Optical Rotation. 3-Substituted 1-Butenes. J. Am. Chem. Soc. 2003, 125, 1888-1896.

(57) Lahiri, P.; Wiberg, K. B.; Vaccaro, P. H. A Tale of Two Carenes: Intrinsic Optical Activity and Large-Amplitude Nuclear Displacement. J. Phys. Chem. A 2012, 116, 9516-9533.

(58) Lahiri, P.; Wiberg, K. B.; Vaccaro, P. H. Intrinsic Optical Activity and Conformational Flexibility: The Role of SizeDependent Ring Morphology in Model Cycloketones. J. Phys. Chem. A 2013, 117, 12382-12400.

(59) Müller, T.; Wiberg, K. B.; Vaccaro, P. H.; Cheeseman, J. R.; Frisch, M. J. Cavity ring-down polarimetry (CRDP): theoretical and experimental characterization. $J$. Opt. Soc. Am. B 2002, 19, 125-141.

(60) Müller, T.; Wiberg, K. B.; Vaccaro, P. H. An optical mounting system for cavity ring-down polarimetry. Rev. Sci. Instrum. 2002, 73, 1340-1342.

(61) Wilson, S. M.; Wiberg, K. B.; Cheeseman, J. R.; Frisch, M. J.; Vaccaro, P. H. Nonresonant Optical Activity of Isolated Organic Molecules. J. Phys. Chem. A 2005, 109, 11752-11764.

(62) Lahiri, P.; Wiberg, K. B.; Vaccaro, P. H.; Caricato, M.; Crawford, T. D. Large solvation effect in the optical rotatory dispersion of norbornenone. Angew. Chem. Int. Ed. 2014, 53, 1386-1389.

(63) Kobayashi, R.; Koch, H.; Jørgensen, P. Calculation of frequency-dependent polarizabilities using coupled-cluster response theory. Chem. Phys. Lett. 1994, 219, 3035 .
(64) Buckingham, A. D.; Dunn, M. B. Optical activity of oriented molecules. Journal of the Chemical Society A: Inorganic, Physical, and Theoretical Chemistry 1971, 1988-1991.

(65) Norman, P.; Ruud, K.; Helgaker, T. Density-functional theory calculations of optical rotatory dispersion in the nonresonant and resonant frequency regions. $J$. Chem. Phys. 2004, 120, 5027-5035.

(66) Krykunov, M.; Autschbach, J. Calculation of origin-independent optical rotation tensor components in approximate timedependent density functional theory. $J$. Chem. Phys. 2006, 125.

(67) Lestrange, P. J.; Egidi, F.; Li, X. The consequences of improperly describing oscillator strengths beyond the electric dipole approximation. J. Chem. Phys. 2015, $143,234103$.

(68) Pelloni, S.; Lazzeretti, P. On the determination of the diagonal components of the optical activity tensor in chiral molecules. J. Chem. Phys. 2014, 140, 074105.

(69) Lazzeretti, P. Invariance of molecular response properties under a coordinate translation. Int. J. Quantum Chem. 2014, 114, 1364-1392.

(70) Becke, A. D. Density-functional Thermochemistry. III. The Role of Exact Exchange. J. Chem. Phys. 1993, 98, 56485652 .

(71) Yanai, T.; Tew, D. P.; Handy, N. C. A new hybrid exchange-correlation functional using the Coulomb-attenuating method (CAM-B3LYP). Chem. Phys. Lett. 2004, 393, 51-57.

(72) Aharon, T.; Caricato, M. Compact Basis Sets for Optical Rotation Calculations. J. Chem. Theory Comput. 2020, 16, 44084415. 
(73) Helgaker, T.; Klopper, W.; Koch, H.; Noga, J. Basis-set convergence of correlated calculations on water. J. Chem. Phys. 1997, 106, 9639-9646.

(74) Frisch, M. J.; Trucks, G. W.; Schlegel, H. B.; Scuseria, G. E.; Robb, M. A.; Cheeseman, J. R.; Scalmani, G.; Barone, V.; Petersson, G. A.; Nakatsuji, H. et al. Gaussian Development Version Revision J.11. 2018.

(75) Moore II, B.; Srebro, M.; Autschbach, J. Analysis of Optical Activity in Terms of Bonds and Lone-Pairs: The Exceptionally Large Optical Rotation of Norbornenone. J. Chem. Theory Comput. 2012, 8, 43364346 .

(76) Srebro, M.; Autschbach, J. Tuned rangeseparated time-dependent density functional theory applied to optical rotation. J. Chem. Theory Comput. 2012, 8, 245256.

(77) Tawada, Y.; Tsuneda, T.; Yanagisawa, S.; Yanai, T.; Hirao, K. A long-rangecorrected time-dependent density functional theory. J. Chem. Phys. 2004, 120, 8425-8433. 